\title{
ROBERT A. WOLAK
}

\section{Foliated and associated geometric structures on foliated manifolds}

\author{
Annales de la faculté des sciences de Toulouse $5^{e}$ série, tome 10, \\ no 3 (1989), p. 337-360 \\ <http://www.numdam.org/item?id=AFST_1989_5_10_3_337_0>
}

(C) Université Paul Sabatier, 1989, tous droits réservés.

L'accès aux archives de la revue «Annales de la faculté des sciences de Toulouse » (http://picard.ups-tlse.fr/ annales/) implique l'accord avec les conditions générales d'utilisation (http://www.numdam.org/conditions). Toute utilisation commerciale ou impression systématique est constitutive d'une infraction pénale. Toute copie ou impression de ce fichier doit contenir la présente mention de copyright.

\section{NumDam}

Article numérisé dans le cadre du programme Numérisation de documents anciens mathématiques http://www.numdam.org/ 


\title{
Foliated and associated geometric structures on foliated manifolds
}

\author{
ROBERT A.WOLAK ${ }^{(1)(2)}$
}

RÉSUME. - Le papier presente une théorie unifiée des structures géométriques sur des variétés feuilletées. Les structures sont classifiées dans trois groupes : feuilletées, transverses et associées. Cette unification du traitement permet de simplifier des démonstrations et de formuler des théorèmes nouveaux sur des feuilletages admettant une structure géométrique.

ABstract. - This paper presents a unified approach to geometric structures on foliated manifolds. Three types of structures are distinguished : foliated, transverse and associated. This unified approach allows to simplify proofs and to obtain new results concerning foliations with additional geometric structure.

In this paper we present a definition of a transverse geometric structure as well as those of foliated and associated structures, and study relations between them. Then we propose a unified approach to these geometric structures. We obtain generalizations of results and simplifications of proofs of R.A. Blumenthal as well as new results on foliations admitting foliated and associated geometric structure. For simplicity's sake all the objects considered are smooth and manifolds are connected, unless otherwise stated.

For another approach to geometric structures on foliated manifolds see P.Molino [26, 28].

In the presentation of transverse structures the author is indebted to Prof.A.Haefliger and his vision of transverse properties. The author had

(1) Departamento de Xeometria e Topoloxia, Facultade de Matematicas, Universidade de Santiago de Compostela, 15705 Santiago de Compostela, Spain

(2) Instytut Matematyki, Uniwersytet Jagielloński, Wl. Reymonta 4, 30-059 Kraków, Poland 
the privilege of discussing these problems with him. In fact, without his comments the definition would have been more restrictive than the one presented in this paper. The author would like to express his gratitude to Prof. P. Molino who helped him understand better foliated structures and whose treatment of foliated G-structures was an inspiration.

During the preparation of this paper the author was a guest of Institut d'Estudis Catalans, Centre de Recerca Matematica in Bellaterra (Barcelona).

\section{Transverse structures}

Let $(M, \mathcal{F})$ be a foliated manifold of dimension $\mathrm{n}$ whose foliation $\mathcal{F}$ is of codimension $q$. The foliation $\mathcal{F}$ is said to be modelled on a $q$-manifold $N_{0}$ if it is defined by a cocycle $\mathcal{U}=\left\{U_{i}, f_{i}, g_{i j}\right\}_{I}$ modelled on $N_{0}$, i.e.

1. $\left\{U_{i}\right\}$ is an open covering of $M$,

2. $f_{i}: U_{i} \longrightarrow N_{0}$ are submersions with connected fibres, and $g_{i j} f_{j}=f_{i}$ on $U_{i} \cap U_{j}$

The $q$-manifold $N=\amalg N_{i}, N_{i}=f_{i}\left(U_{i}\right)$, is called the transverse manifold associated to the cocycle $\mathcal{U}$ and the pseudogroup $\mathcal{H}$ of local diffeomorphisms of $N$ generated by $g_{i j}$ the holonomy pseudogroup representative on $N$ (associated to the cocycle $\mathcal{U}$ ). $N$ is a complete transverse manifold. The equivalence class of $\mathcal{H}$ we call the holonomy pseudogroup of $\mathcal{F}$ (or $(M, \mathcal{F})$ ). In what follows, we assume that $\mathcal{F}$ is defined by a cocycle $\mathcal{U}$ and we denote by $N$ and $\mathcal{H}$ the transverse manifold and holonomy pseudogroup associated to $\mathcal{U}$, respectively. It is not difficult to check that different cocycles defining the same foliation provide us with equivalent holonomy pseudogroups, $\mathrm{cf}$. [16]. In general the converse is not true.

Example 1. - Let $\mathcal{F}$ be a transversely affine foliation whose developing mapping is surjective, cf. [14]. Its holonomy pseudogroup has a representative $\mathcal{H}_{\Gamma}$ - the pseudogroup defined by the action of the affine holonomy group $\Gamma$ on $R^{q}$. If the fibres of the developing mapping are not connected and some connected components correspond to different leaves of $\mathcal{F}$, then $R^{q}$ cannot be a complete transverse manifold of the foliation $\mathcal{F}$, and $\mathcal{H}_{\Gamma}$ is not a representative of the holonomy pseudogroup associated to any cocycle defining the foliation $\mathcal{F}$. As an example of such a foliation we can take the one-dimensional Hopf foliation of $S^{2} \times S^{1}$. 
The precise statement of the "converse" result is best expressed using the notion of a $\mathcal{K}$-foliation, cf. [15]. Let $\mathcal{K}$ be any pseudogroup of local diffeomorphisms on a $q$-manifold $N_{0}$. A $\mathcal{K}$-foliation is a foliation defined by a cocycle $\mathcal{U}$ modelled on $N_{0}$ with $g_{i j}$ being elements of the pseudogroup $\mathcal{K}$. Then the holonomy pseudogroup associated to $\mathcal{U}$ is equivalent to a subpseudogroup of $\mathcal{K}$. With this in mind we have the following.

Leмma 1.- Let $\mathcal{F}$ be a foliation defined by a cocycle $\mathcal{U}$ and let $\left(\mathcal{H}^{\prime}, N^{\prime}\right)$ be a pseudogroup equivalent to $(\mathcal{H}, N)$. Then $\mathcal{F}$ is an $\mathcal{H}^{\prime}$-foliation.

To introduce the definition of a transverse geometric structure we use the notion of a natural bundle, cf. [32].

Definition 1.- Let $N$ be a transverse manifold of the foliation $\mathcal{F}$. An $\mathcal{H}$-invariant subbundle $E$ of a natural fibre bundle $F(N)$ is called a transverse geometric structure of the foliation $\mathcal{F}$.

It is not difficult to see that the definition does not depend on the choice of a cocycle $\mathcal{U}$ defining the foliation (i.e. on the choice of a transverse manifold and holonomy pseudogroup). Let $\left(\mathcal{H}^{\prime}, N^{\prime}\right)$ be a pseudogroup equivalent to $(\mathcal{H}, N)$ and let $\Phi=\left(\phi_{\alpha}\right)$ be the equivalence. Then the subbundle $E_{\Phi}=\left\{v^{\prime} \in\right.$ $\left.F\left(N^{\prime}\right): v^{\prime}=F\left(\phi_{\alpha}\right)(v), v \in E\right\}$ (resp. $E_{\Phi}=\left\{v^{\prime} \in F\left(N^{\prime}\right): F\left(\phi_{\alpha}\right)\left(v^{\prime}\right) \in E\right\}$ for a contravariant functor) is an $\mathcal{H}^{\prime}$-invariant subbundle of $F\left(N^{\prime}\right)$ which is locally isomorphic to the subbundle $E$. Therefore, it is possible to talk about holonomy invariant subbundles on the transverse manifold. Moreover, when solving a particular problem, we can choose a cocycle making our foliation a $\mathcal{K}$-foliation for a suitable pseudogroup $\mathcal{K}$.

Example 2. - 1. Let $L$ be the functor associating to a manifold its bundle of linear frames. Then any $\mathcal{H}$-invariant $G$-reduction of $L(N)$ is a transverse geometric structure. Such a foliation is called a $G$-foliation, cf. $[19,24,33]$.

2. Any $\mathcal{H}$-invariant section of a fibre bundle $F(N)$ is a transverse geometric structure. Thus, if we take the functor of the tangent bundle we get holonomy invariant vector fields on the transverse manifold. For the contravariant functor of the cotangent bundle we get $\mathcal{H}$-invariant 1 -forms and as $\mathcal{H}$-invariant sections of the tensor products of these bundles(functors) we get $\mathcal{H}$-invariant tensor fields.

Using a suitable associated fibre bundle to the bundle of linear frames (such functors are natural fibre bundles), we obtain that any $\mathcal{H}$-invariant linear connection in an $\mathcal{H}$-invariant $G$-structure is a transverse geometric 
structure. Such foliations we have called $\nabla-G$-foliations, cf. [39]. Riemannian and transversely affine foliations belong to this class.

We obtain holonomy invariant connections of higher order or Cartan connections as holonomy invariant sections of associated fibre bundles to the bundle of frames of higher order. Such connections exist for transversely conformal or transversely projective foliations, cf. [8].

3. Let $\mathcal{F}$ be a $\mathcal{K}$-foliation. If $\mathcal{K}$ is a Lie pseudogroup, then the subbundle of $r$-jets from the definition of a Lie pseudogroup, cf. [34], is a transverse geometric structure. Foliations admitting foliated systems of differential equations are of this type, cf. [42].

Many geometric constructions provide useful ways of constructing new transverse geometric structures. In fact, the holonomy pseudogroup is a pseudogroup of local automorphisms of a given transverse geometric structure. Any geometric object related to this structure and invariant under the action of the pseudogroup of local automorphisms of the initial structure is itself a transverse geometric structure. For example, take a holonomy invariant $G$-structure $B(N, G)$. Then its prolongations and their structure tensors are also holonomy invariant, cf. $[20,35]$. If $\nabla$ is a holonomy invariant connection in $B(N, G)$, then its torsion and curvature tensor fields $T$ and $R$, respectively, are holonomy invariant as well as $\nabla^{k} T$ and $\nabla^{k} R$.

\section{Foliated structures}

A foliated geometric structure, intuitively, is a geometric stucture on a foliated manifold which is "locally constant" along the leaves; for example, in local adapted coordinates it can be expressed in transverse coordinates only. It is the case of a foliated vector field or the Riemannian metric induced on the normal bundle by a bundle-like Riemannian metric. To present the definition of a foliated structure we need the following.

Let $\underline{F o l}_{q}$ be the category of foliated manifolds with codimension $q$ foliations. Global mappings which preserve foliations and which are transverse to them are the morphisms in this category, i.e.

$$
f \in M o r\left(\left(M_{1}, \mathcal{F}_{1}\right),\left(M_{2}, \mathcal{F}_{2}\right)\right) \text { iff } d f\left(T \mathcal{F}_{1}\right) \subset T \mathcal{F}_{2} \text { and } f^{*} \mathcal{F}_{2}=\mathcal{F}_{1} \text {. }
$$

DEFINITION 2.- A covariant (resp. contravariant) functor $F$ on the category $\mathrm{Fol}_{q}$ into the category of locally trivial fibre bundles and their fibre mappings is called a foliated natural bundle if the following conditions are satisfied : 
Foliated and associated geometric structures on foliated manifolds

i) for any foliated manifold $(M, \mathcal{F}), F(M, \mathcal{F})$ is a locally trivial fibre bundle over $M$;

ii) let $f \in \operatorname{Mor}\left(\left(M_{1}, \mathcal{F}_{1}\right),\left(M_{2}, \mathcal{F}_{2}\right)\right)$. Then the fibre mapping $F(f)$ has the following properties :

a) covariant case :

- $F(f)$ covers $f$, i.e. the following diagram is commutative :

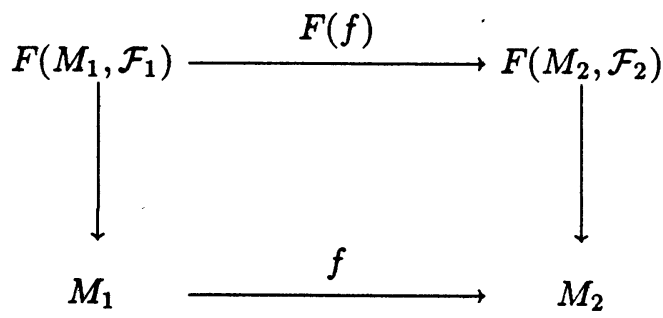

- for any point $x$ of $M_{1}$, the mapping $F(f)_{x}:\left(M_{1}, \mathcal{F}_{1}\right)_{x} \longrightarrow\left(M_{2}, \mathcal{F}_{2}\right)_{f(x)}$ is a diffeomorphism;

b) contravariant case :

- for any point $x$ of $M_{1}$, the mapping $F(f)_{x}:\left(M_{2}, \mathcal{F}_{2}\right)_{f(x)} \longrightarrow\left(M_{1}, \mathcal{F}_{1}\right)_{x}$ is a diffeomorphism,

- the following diagram is commutative :

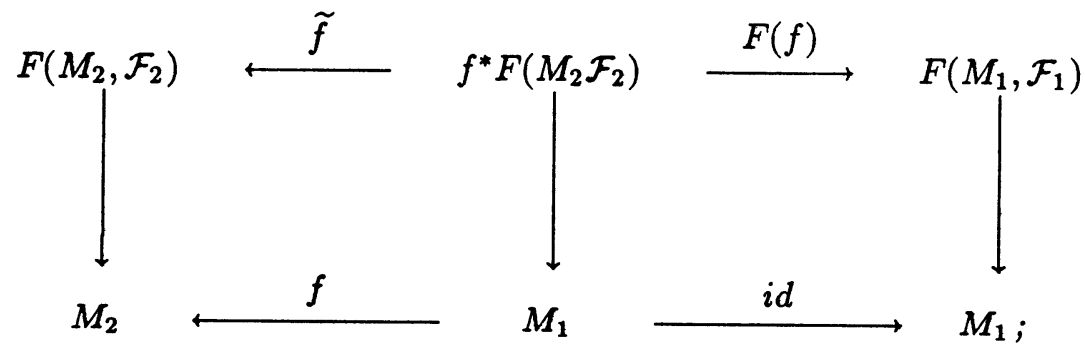

iii) the functor $F$ is regular.

Remark. - In [41] we have called such functors transverse natural bundles. 
Example 3.- The following functors are foliated natural bundles, cf. $[23,41]$.

- normal bundle,

- normal bundle of order $r$,

- bundle of transverse linear frames,

- bundle of transverse $r$-frames,

- bundle of transverse $(p, r)$-velocities,

- bundle of transverse $A$-points,

- dual bundle of the normal bundle,

- any tensor product of these bundles,

- any associated fibre bundle to the bundles of transverse frames.

The use of the adjective "foliated" is best explained by the following. Let $F$ be any covariant foliated natural bundle functor. If the foliation $\mathcal{F}$ is defined by a cocycle $\mathcal{U}$ then $\left\{F\left(U_{i}\right), F\left(f_{i}\right), F\left(g_{i j}\right)\right\}_{I}$ is a cocycle defining a foliation $\mathcal{F}_{F}$ on the total space of the bundle $F(M, \mathcal{F})$. The leaves of the foliation $\mathcal{F}_{F}$ are coverings of leaves of $\mathcal{F}$. In the contravariant case we define this cocycle as follows :

We start with the commutative diagram :

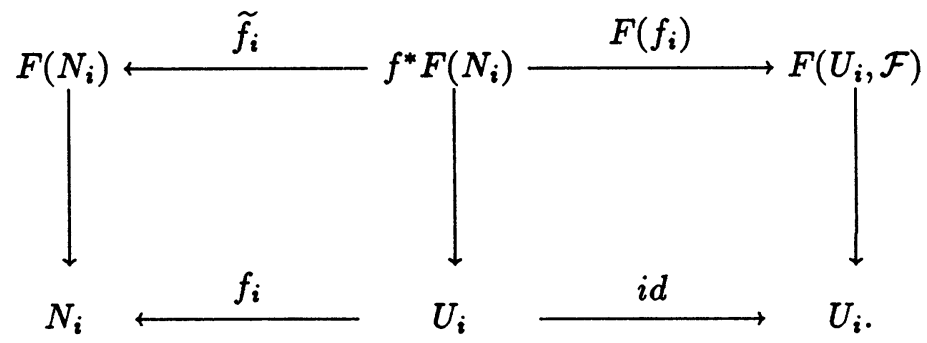

Then take $\widetilde{F}\left(f_{i}\right): F\left(U_{i}, \mathcal{F}\right) \longrightarrow F\left(N_{i}\right)$ equal to $\tilde{f}_{i} F\left(f_{i}\right)^{-1}$ and $\widetilde{g}_{i j}=F\left(g_{j i}\right)$. Indeed, $\left\{F\left(U_{i}, \mathcal{F}\right), \widetilde{F}\left(f_{i}\right), \widetilde{g}_{i j}\right\}_{I}$ is a cocycle defining a foliation $\mathcal{F}_{F}$ on the total space of the bundle $F(M, \mathcal{F})$ whose leaves are coverings of leaves of $\mathcal{F}$. It is not difficult to verify that to equivalent cocycles defining the foliation $\mathcal{F}$ correspond equivalent cocycles on the total space of $F(M, \mathcal{F})$. Therefore the foliation $\mathcal{F}_{F}$ does not depend on the choice of a cocycle defining $\mathcal{F}$.

One can easily verify that any foliated natural bundle is determined by its values on $q$-manifolds. In fact, the bundle $\mathcal{F}_{\boldsymbol{U}}=\amalg f_{i}^{*} F\left(N_{i}\right) / \sim$ where $(x, i, v) \sim\left(x^{\prime}, j, v^{\prime}\right)$ iff $x=x^{\prime}$ and $v^{\prime}=F\left(g_{j i}\right)(v)$ (resp. $v=F\left(g_{j i}\right)\left(v^{\prime}\right)$ in the 
contravariant case), is a well-defined locally trivial fibre bundle over M. Two equivalent cocycles define isomorphic bundles. Moreover this isomorphism preserves the natural foliations (locally defined by projections $f_{i}^{*} F\left(N_{i}\right) \longrightarrow$ $F\left(N_{i}\right)$ ) of these two bundles. The bundle $F(M, \mathcal{F})$ is isomorphic to $F_{\mathcal{U}}$ and this isomorphism is foliation preserving. Therefore any natural functor can be uniquely extended to a foliated natural one.

We have noticed that any foliated natural bundle $F(M, \mathcal{F})$ admits a foliation $\mathcal{F}_{F}$ of the same dimension as $\mathcal{F}$. Therefore we can talk foliated subbundles of $F(M, \mathcal{F})$, i.e. those whose total space is saturated for $\mathcal{F}_{F}$, cf. $[18,24,29]$. This leads us to the following definition of a foliated geometric structure.

Definition 3.- $A$ foliated subbundle $E$ of a foliated natural bundle $F(M, \mathcal{F})$ is called a foliated geometric structure.

The previous considerations ensure that these foliated subbundles are in one-to-one correspondence with holonomy invariant subbundles of the corresponding natural bundle on the transverse manifold. Therefore foliated geometric structures are in one-to-one correspondence with transverse ones.

This correspondence can be presented in the following dictionary :

\section{foliated}

normal bundle of order $r$

bundle of transverse $(\mathrm{p}, \mathrm{r})$-velocities

bundle of transverse A-points

foliated natural bundle

foliated vector field

base-like $r$-form (on the normal bundle)

foliated tensor field of type $(p, r)$

foliated G-structure

fundamental form of " "

structure tensor of " "

$k$ th prolongation of " "

transversely projectable $G$-connection

torsion tensor of " "

curvature tensor of " "

bundle-like metric (on the normal bundle) transverse sectional curvature

foliated Cartan connection

curvature of " " "

\section{Dictionary}

\section{holonomy invariant}

tangent bundle of order $r$

bundle of $(\mathrm{p}, \mathrm{r})$-velocities

bundle of A-points

natural bundle

vector field

r-form

tensor field of type $(\mathrm{p}, \mathrm{r})$

G-structure

fundamental form of "

structure tensor of "

$k$ th prolongation of "

$G$-connection

torsion tensor of "

curvature tensor of "

Riemannian metric

sectional curvature

Cartan connection

curvature of " " 
In [25] P.Molino considers foliations admitting transversely projectable connections. The correspondence between structure tensors and prolongations have been proved in $[24,40]$. Foliated Cartan connections were introduced by M.Takeuchi in [36] and R.A.Blumenthal in [8] and transverse sectional curvature in [7].

The dictionary reduces the study of "foliated" problems to holonomy invariant ones and sometimes to the right choice of a cocycle defining the foliation. This, owing to Lemma 1 , is equivalent to a good choice of a representative of the holonomy pseudogroup.

Let us analyze the results of R.A.Blumenthal presented in $[3,4,5,6,8]$. In $[3,4,5,6]$ he considers foliations admitting a transversely projectable connection whose curvature and torsion tensors have some additional properties. These properties, the connection being transversely projectable, can be read as the properties of the curvature and torsion tensors, respectively, of the corresponding holonomy invariant connection on the transverse manifold. In Blumenthal's case well-known theorems ensure that we can choose a very good representative of the holonomy pseudogroup. It is precisely what Blumenthal did in each case. In fact, as a holonomy pseudogroup representative we can take a subpseudogroup of the pseudogroup obtained as the localization of an quasi-analytical action of a Lie group $K$ on a connected simplyconnected manifold $N_{0}$. Therefore, our foliation is an $\left(N_{0}, K\right)$-structure and a developable foliation, $\mathrm{cf}$. $[12,14,37]$. The completeness assumptions ensure that the developing mapping is a locally trivial fibre bundle, and this fact yields the most important results.

At the basis of these four papers is the following scheme :

- Realize that the considered geometric objects on the foliated manifold are foliated and pass to the holonomy invariant ones.

- Using theorems on local equivalence of geometric structures choose a good representative of the holonomy pseudogroup.

- Check that any element of the holonomy pseudogroup can be uniquely extended to a global one.

- Verify that "completeness" assumptions ensure that the developing mapping is a locally trivial fibre bundle; for example the foliated differential equation defined by the foliated geometric object is a transversely complete foliated differential equation and it projects onto a differential equation on the developing image, $\mathrm{cf}$. Theorem 1 of [42].

The same scheme has been applied to foliations admitting Cartan connections in [8]. 
Having formulated our proof scheme we can prove the results contained in $[3,4,5,6]$ quite easily. We only need some results on local equivalence of reductive and locally symmetric spaces, see for example $[21,38]$. In the case of Cartan connections we should take into account Lemma 11.10 and Proposition 11.1 of [31]. Then the equation of the geodesic of the transversely projectable connection is a tranversely complete foliated differential equation corresponding to the equation of the geodesic of the connection of the transverse manifold. Therefore the developing mapping must be locally trivial.

The theory of foliated Cartan connections is very similar to its classical counterpart, cf. [31]. Let us restrict our attention to $G$-structures of second order modelled on a semi-simple homogeneous space. Let $B(M, G ; \mathcal{F})$ be such a foliated $G$-structure. Its total space $B$ is foliated by a foliation $\mathcal{F}_{B}$. As in the case of 1 st order $G$-structures foliated Cartan connections do not always exist. The standard construction ( using the partition of unity and local existence ) ensures the existence of basic Cartan connections, i.e. 1forms on $B$ with values in $\operatorname{Lie}(L)=\underline{l}$ vanishing on vectors tangent to the foliation $\mathcal{F}_{B}$. It is also possible to introduce the notion of basic admissible Cartan connections. The vanishing of the Spencer cohomology group $H^{2,1}(\underline{l})$ ensures the existence of a foliated Cartan connection, cf. Theorem 1.1 of [36]. In fact, the vanishing of this cohomology group makes sure that in the corresponding $G$-structure on the transverse manifold there exists the normal Cartan connection and that this connection is holonomy invariant. Thus it defines a foliated Cartan connection in $B(M, G ; \mathcal{F})$ which we call the normal foliated Cartan connection of this $G$-structure. Next one can define its Weyl tensor, and it is not difficult to verify that it is a foliated tensor. In some cases its vanishing ensures that the model $G$-structure is flat. This leads us to formulate the following theorem which is a generalization of Theorem 2 of [8].

Theorem 1.- Let $B(M, G ; \mathcal{F})$ be a foliated $G$-structure of second order modelled on a semi-simple flat homogeneous space $L / L_{0}$ such that $H^{2,1}(\underline{l})$ $=H^{2,2}(\underline{l})=H^{1,1}(\underline{l})=0$. If the normal foliated Cartan connection of this structure is complete, and the Weyl tensor of $B(M, G ; \mathcal{F})$ vanishes, then $\mathcal{F}$ is an $\left(L / L_{0}, L\right)$-structure and the developing mapping $h: \widetilde{M} \longrightarrow \widetilde{L / L_{0}}($ $\widetilde{L / L_{0}}$ is the universal covering space of $L / L_{0}$ ) is a locally trivial fibre bundle whose fibres are the leaves of the lifted foliation $\tilde{\mathcal{F}}$. 
Proof.- We follow our proof scheme.7Since the Weyl tensor is foliated, the Weyl tensor of the corresponding $G$-structure on the transverse manifold vanishes. Ochai's theorem (cf. [31] Theorem 12.1) ensures that the normal Cartan connection is flat. Then according to Proposition 11.1 and Lemma 11.10 of the same paper the foliation $\mathcal{F}$ is an $\left(L / L_{0}, L\right)$-structure. The completeness of the normal foliated Cartan connection means that the foliation $\mathcal{F}_{B}$ is a complete transversely parallelisable foliation, cf. [8], and that the equation of the geodesic of this connection is complete. This first fact ensures that the holonomy coverings of leaves of $\mathcal{F}$ are diffeomorphic, and the second that the developing mapping is a locally trivial fibre bundle cf. Theorem 1 of [42].

Let us provide some more background material for our "proof scheme". Local equivalence of geometric structures have been studied for many years. The best account can be found in [1], see also [27]. Geometers looked for a set of invariants of $\mathcal{K}$-structures ( $\mathcal{K}$ a Lie pseudogroup) which would ensure that any two $\mathcal{K}$-structures having the same invariants are locally equivalent. Having defined the structure tensors, it was necessary to determine whether the formal integrability (i.e. all structure tensors vanish) is equivalent to the integrability (i.e. the $\mathcal{K}$-structure is locally equivalent to the corresponding canonical flat structure on $R^{n}$ ). It is true for any $G$-structure, as well as for many other $\mathcal{K}$-structures, cf. [1]. We can say a little more about $G$ structures of finite type : two $G$-structures with the same constant structure tensors are locally equivalent, cf. $[20,35]$. Therefore transverse $G$-structures of foliated $G$-structures of finite type with the same constant structure tensors are locally equivalent. Thus any foliated $G$-structure of finite type with vanishing structure tensors can be modelled on the canonical flat $G$-structure of $R^{q}$. The group of automorphisms of such a $G$-structure $B\left(N_{0}, G\right)$ of finite type is a Lie group, and its elements are determined by their finite jets, cf. [20]. Therefore, this group acts quasi-analytically on the manifold $N_{0}$.

Having chosen a model $(\mathcal{K}, N)$ of the transverse structure of the foliation $\mathcal{F}$, with $N$ a connected manifold, we would like to know whether the pseudogroup $\mathcal{K}$ is generated by a group, i.e. whether any element of $\mathcal{K}$ can be uniquely extended to a global diffeomorphism of $N$. It is a very well known problem, and there are many theorems of this type. We have already used some of them. They are based on the following principle :

There exists a fibre bundle $B(N)$ over $N$ whose total space is parallelisable and which has the following properties : 
- the vector space spanned by vector fields of the parallelism consists of complete vector fields,

- the group generated by the flows of these vector fields acts effectively (i.e. $f(x)=x$ implies $f=i d$,

- elements of the pseudogroup $\mathcal{K}$ lift to local diffeomorphisms of the total space of $B(N)$ which commute with the parallelism,

- any local diffeomorphism commuting with the parallelism is, locally, the lift of an element of $\mathcal{K}$.

Then, of course, any element of $\mathcal{K}$ can be uniquely extended to a global diffeomorphism of $N$. In some cases it is possible to verify directly that the pseudogroup of automorphisms of a given geometric structure has this extension property.

In general, it is easier to solve the infinitesimal version of this problem, (as it only concerns solutions of systems of linear differential equations) : can any local $\mathcal{K}$-vector field be extended to a global one?, cf. [30,22,2]. Having a positive answer to this question does not solve the extension problem for the pseudogroup $\mathcal{K}$. First of all, we have to know whether any element of $\mathcal{K}$, at least locally, can be represented as the composition of a finite number of elements of flows of $\mathcal{K}$-vector fields and whether global $\mathcal{K}$-vector fields are complete. The first one have been studied thoroughly in the framework of Lie pseudogroups and we have a definite answer, cf. [34] Propositions 3.6 and 3.7. The second one is just the question whether a certain differential equation has global solutions.

The above considerations lead to several interesting results concerning $G$-foliations. Let us recall that a $G$-foliation with the group $G$ of type $k$ is transversely complete if for a given choice of a subbundle $Q$ supplementary to $\mathcal{F}$ the transverse parallelism of the foliation $\mathcal{F}_{k}$ of the total space of $B^{(k-1)}(M, G ; \mathcal{F})$ is complete, $\mathrm{cf}$. $[7,40]$.

TheOREM 2.- Let $N$ be a simply connected compact analytic manifold with an analytic $G$-structure $B(N, G)$ of finite type. Let $\mathcal{H}$ be a connected regular pseudogroup of local analytic automorphisms of $B(N, G)$. Then any $\mathcal{H}$-foliation $\mathcal{F}$ is developable. Moreover, if the $G$-foliation $\mathcal{F}$ is transversely complete, then the developing mapping is a locally trivial fibre bundle whose fibres are the leaves of the lifted foliation.

Proof.- Our proof scheme takes care of everything but the fact that the foliation $\mathcal{F}$ is an $(N, K)$-structure. Proposition 3.6 or 3.7 of [34] ensures 
that any element of the pseudogroup $\mathcal{H}$, locally, can be represented as the composition of a finite number of local diffeomorphisms from flows of $\mathcal{H}$ vector fields. The theorem of Amores, cf. [2], makes sure that any local (analytic) infinitesimal automorphism of the $G$-structure $B(N, G)$ can be extended to a global one, and as the manifold $N$ is compact, these vector fields are complete. Thus $\mathcal{F}$ is an $(N, \operatorname{Aut}(B(N, G))$-structure. The local triviality of the developing mapping results from the fact that the complete TUSP foliated system of differential equations on $(M, \mathcal{F})$ defined by the transverse parallelism of $\mathcal{F}_{k}$ projects via the developing mapping onto a system of differential equations defined by the parallelism of $B^{(k-1)}(N, G)$.

The results of A.Y.Ledger and M.Obata lead us to the formulation of the following theorem, cf. [22].

THEOREM 3. - Let $(M, \mathcal{F})$ be a conformal but non-Riemannian transversely analytic foliation of codimension $q(q>2)$. Then :

1. Let the foliation $\mathcal{F}$ be modelled on a compact Riemannian analytic manifold with finite fundamental group whose pseudogroup $\mathcal{C}$ of local conformal transformations is a regular Lie pseudogroup. If the holonomy pseudogroup of $\mathcal{F}$ is contained in the connected component of id in $\mathcal{C}$ then the foliation $\mathcal{F}$ is developable and the developing mapping is into the $q$-sphere $S^{q}$.

2. If, additionally, the foliation $\mathcal{F}$ is transversely complete, then the developing mapping is a locally trivial fibre bundle with fibres being leaves of the lifted foliation.

Taking into account the results of A.Y.Ledger and M.Obata the proof of this theorem is the same as that of Theorem 2.

THEOREM 4. - Let $(M, \mathcal{F})$ be a G-foliation of type 1 with vanishing structure tensors. If the holonomy pseudogroup $\mathcal{H}$ on the transverse manifold $N$ is contained in the connected component of id of the pseudogroup of local automorphisms of the $G$-structure $B(N, G)$, then the foliation $\mathcal{F}$ is developable. Moreover, if $\mathcal{F}$ is transversely complete, then the developing mapping is a locally trivial fibre bundle over $R^{q}$ with fibres being the leaves of the lifted foliation.

Proof.-The vanishing of the structure tensors of the foliated $G$ structure $B(M, G ; \mathcal{F})$ ensures that the structure tensors of $B(N, G)$ also vanish. Therefore the $G$-structure $B(N, G)$ is integrable, cf. $[20,35]$, and the foliation $\mathcal{F}$ is modelled on the canonical flat $G$-structure of $R^{q}$. Since 
the group $G$ is of type 1 , only the vector fields of the form $\sum_{j=1}^{q} a_{i}^{j} \partial_{j}$ or $\sum_{i, j=1}^{q} a_{i}^{j} x_{j} \partial_{j}\left(\left(a_{j}^{i}\right) \in \operatorname{Lie}(G)\right)$ are infinitesimal automorphisms of this flat $G$-structure, and any local infinitesimal automorphism can be extended to a global one. It is not difficult to see that these vector fields are complete. The rest of the proof is standard.

Using our proof scheme we can produce some other theorems of this kind. For example, by imposing conditions on the transverse sectional curvature of a Riemannian foliation we obtain :

THEOREM 5.- Let $(M, \mathcal{F}, g)$ be a complete Riemannian foliation. If the transverse sectional curvature is constant or depends only on the point of the manifold $M$, then the foliation $\mathcal{F}$ is developable, and the developing mapping is a locally trivial fibre bundle over $R^{q}, S^{q}$ or $H^{q}$ with fibres being the leaves of the lifted foliation.

Proof.- The second condition ensures that the transverse sectional curvature is constant (a foliated version of Schur lemma which can be easily proved or deduced from the original one). Then the theorem on local isometries of such Riemannian manifolds, cf. [21,38], and our proof scheme take care of the rest.

There are many applications of these theorems. We shall give only some of them, see also $[3,4,6,8]$. We assume that $\mathcal{F}$ is transversely complete and that the assumptions of one of the theorems are satisfied.

CoROllary 1. - Let $N$ be a contractible $q$-manifold and $K$ a Lie group acting quasi-analytically on it. If the foliation $\mathcal{F}$ is an $(N, K)$-structure then the universal covering space $\widetilde{M}$ of the manifold $M$ is the product $\widetilde{L} \times N$, where $\widetilde{L}$ is the common universal covering space of leaves of the foliation $\mathcal{F}$.

Proof.- In this case the developing mapping is a trivial bundle.

COROLlaRY 2.- On a compact manifold with a finite fundamental group there are no G-foliations satisfying the assumption of Theorem 4.

Proof. - In this case, the universal covering space would be both compact and diffeomorphic to $\widetilde{L} \times R^{q}$; contradiction.

CoROllaRY 3.- Let $\Phi$ be a flow on a compact manifold with finite fundamental group. If the flow $\Phi$ admits a foliated structure satisfying the assumptions of one of the theorems then its leaves have finite holonomy. If all its orbits are closed then $\Phi$ is a Riemannian flow. 
Proof. - Assume the contrary. Let $L$ be a leaf with infinite holonomy. Its holonomy covering cannot be compact. Thus the developing mapping is a locally trivial fibre bundle whose total space is compact but whose fibres are diffeomorphic to $R$. Contradition. Then the second assertion follows from [13], see also [44].

It is worth to mention that the assumption of "completeness" of a foliated structure does not translate itself easily into the language of holonomy invariant properties. The only really succesful case is the Riemannian one where A.Haefliger has introduced the notion of a complete pseudogroup of local isometries, cf. [16,17]. For other attempts see [43].

\section{Associated structures}

On a foliated manifold there are other structures than foliated ones. Let us consider the following two examples.

Example 4.- A foliated vector field, which is a foliated section of the normal bundle, is a foliated structure. On the other hand it is an equivalence class of global infitesimal automorphisms of the foliation $\mathcal{F}$. Such a global infinitesimal automorphism is a foliated section of the tangent bundle $T M$ with the foliation $\mathcal{F}_{T M}$ defined by the atlas of $T M$ associated to the adapted atlas of $(M, \mathcal{F})$. The foliation $\mathcal{F}_{T M}$ has codimension $2 q$. Its leaves project onto the leaves of $\mathcal{F}$ and the natural projection $p_{N}: T M \longrightarrow N(M ; \mathcal{F})$ is a morphism in $\underline{\mathrm{Fol}}_{2 q}$.

Example 5. - Let $L_{\mathcal{F}}(M)$ be the bundle of linear frames adapted to $\mathcal{F}$, i.e. $\left(v_{1}, \ldots v_{n}\right) \in L_{\mathcal{F}}(M)_{x}$ iff $v_{1}, \ldots v_{n-q}$ span the subspace tangent to $\mathcal{F}$ at the point $x$. There is a natural mapping $p_{L}$ from this bundle into the bundle of transverse linear frames $L(M ; \mathcal{F})$ assigning to any frame $\left(v_{1}, \ldots v_{n}\right)$ the frame $\left(\widetilde{v}_{1}, \ldots \widetilde{v}_{q}\right)$ corresponding to the vectors $v_{n-q+1}, \ldots v_{n}$. As in the previous example the adapted atlas of $\mathcal{F}$ defines a foliation $\mathcal{F}_{L}$ of codimension $q+q^{2}$ on the total space of $L_{\mathcal{F}}(M)$. The leaves of $\mathcal{F}_{L}$ project onto the leaves of $\mathcal{F}$. The mapping $p_{L}$ is a morphism in the category $\underline{F o l}_{q^{2}+q}$.

In the above examples we have presented two structures which are not foliated in our sense, but which are closely related to the structure of a foliated manifold. These two structure are, what we call, associated geometric structures. Now we are going to present a formal definition.

Let $\mathrm{Fol}_{q}^{*}$ be a category of foliated manifolds with foliations of codimension $q$. The morphisms in this category are the following : 
$f \in \operatorname{Mor}\left(\left(M_{1}, \mathcal{F}_{1}\right),\left(M_{2}, \mathcal{F}_{2}\right)\right)$ iff

1. $\operatorname{dim} M_{1}=\operatorname{dim} M_{2}$, then $f: M_{1} \longrightarrow M_{2}$ is an embedding and $f^{*} \mathcal{F}_{2}=\mathcal{F}_{1}$,

2. $\operatorname{dim} M_{1}>\operatorname{dim} M_{2}$, then $\operatorname{dim} M_{2}=q, \mathcal{F}_{2}$ is the foliation by points and $f: M_{1} \longrightarrow M_{2}$ is a submersion defining $\mathcal{F}_{1}$.

DEFINITION 4.- An associated natural bundle is a functor defined on $\mathrm{Fol}_{q}^{*}$ with values in the category of locally trivial fibre bundles such that:

i) the bundle $F(M, \mathcal{F})$ is a locally trivial bundle over $M$;

ii) for any morphism $f \in \operatorname{Mor}\left(\left(M_{1}, \mathcal{F}_{1}\right),\left(M_{2}, \mathcal{F}_{2}\right)\right), F(f)$ is a bundle mapping such that :

a) covariant case : the diagram

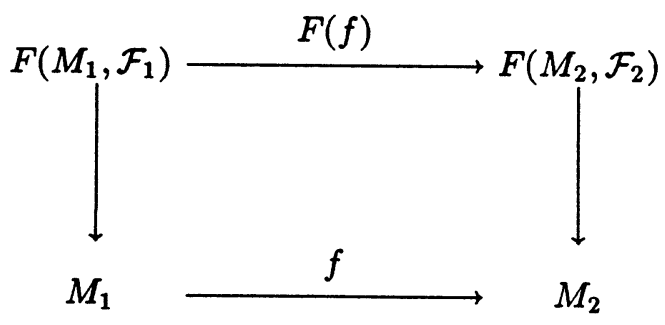

is commutative. Moreover, for any $x \in M_{1}$, if $\operatorname{dim} M_{1}=\operatorname{dim} M_{2}$ the mapping $F(f)_{x}: F\left(M_{1}, \mathcal{F}_{1}\right)_{x} \rightarrow F\left(M_{2}, \mathcal{F}_{2}\right)_{f(x)}$ is a diffeomorphism, and if dimM $M_{1}>$ $\operatorname{dim} M_{2}=q$ then this mapping is a surjective submersion;

b) contravariant case : the diagram

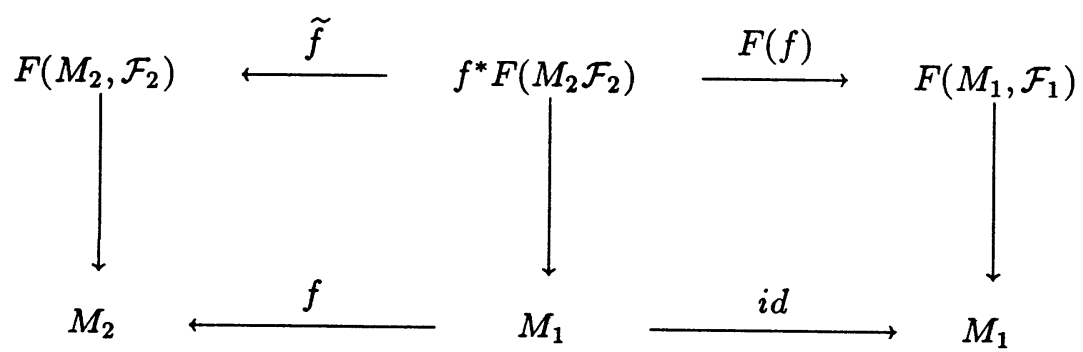

is commutative, where $\tilde{f}$ is the natural projection induced by $f$. Moreover, for any points $y \in M_{2}, x \in f^{-1}(y)$, if dim $M_{1}=\operatorname{dim}_{2}$ the mapping $F(f)_{y}: F\left(M_{2}, \mathcal{F}_{2}\right)_{y} \rightarrow F\left(M_{1}, \mathcal{F}_{1}\right)_{x}$ is a diffeomorphism, and if dim $M_{1}>$ $\operatorname{dim}_{2}=q$ then this mapping is an embedding; 
iii) the functor $F$ is regular.

Remark. - If we consider the category $\underline{M a n}_{q}$ as a subcategory of $\underline{F o l}_{q}^{*}$, the restriction of any associated natural bundle functor to this subcategory is a natural bundle functor. In general, it does not seem to be possible to reconstruct an associated natural bundle functor from its values on $\underline{M a n}_{q}$. But owing to the considerations of the previous section, any associated natural bundle defines a foliated natural bundle, e.g. the passage from the bundle of linear frames adapted to the foliation to the bundle of transverse linear frames. Moreover, any foliated natural bundle functor is an associated one.

DEFINITION 5. - Let $f$ be a morphism in Fol $_{q}^{*}$, $f \in \operatorname{Mor}\left(\left(M_{1}, \mathcal{F}_{1}\right),\left(M_{2}, \mathcal{F}_{2}\right)\right)$.

Two subbundles $B_{1}$ of $F\left(M_{1}, \mathcal{F}_{1}\right)$ and $B_{2}$ of $F\left(M_{2}, \mathcal{F}_{2}\right)$ are said to be $f$ related if the fibre mapping $F(f)$ restricted to $B_{1}$ is a surjective submersion onto $B_{2}$ (resp. it is a diffeomorphism of $f^{*} B_{2}$ onto $B_{1}$ in the contravariant case).

Let $\mathcal{U}$ be a cocycle defining the foliation $\mathcal{F}$ modelled on $N$. In the covariant case this cocycle defines a cocycle $\mathcal{U}_{F}$ on the total space of the bundle $F(M, \mathcal{F})$, namely : $\left\{V_{i}, \tilde{f}_{i}, \tilde{g}_{i j}\right\}_{I}$ where $F(M, \mathcal{F}) \mid U_{i}=V_{i}, \tilde{f}_{i}=$ $F\left(f_{i}\right), \tilde{g}_{i j}=F\left(g_{i j}\right)$. The foliation $\mathcal{F}_{F}$ defined by this cocycle (equivalent cocycles of $\mathcal{F}$ give equivalent ones) is not of the same dimension as $\mathcal{F}$ but it projects onto $\mathcal{F}$. The codimension of $\mathcal{F}_{F}$ is equal to $\operatorname{dim} F(N)$. In the contravariant case, we obtain a subbundle $\widetilde{F}(M, \mathcal{F})$ of the fibre bundle $F(M, \mathcal{F})$. Over $U_{i}$, it is isomorphic to $f_{i}^{*} F(N)$ and therefore it is naturally foliated. This subbundle and its foliation $\mathcal{F}_{F}$ does not depend on the choice of the cocycle $\mathcal{U}$. The foliation has codimension equal to $\operatorname{dim} F(N)$ and is of the same dimension as $\mathcal{F}$.

Definition 6. - A foliated subbundle $E$ of $F(M, \mathcal{F})$ (resp. of $\widetilde{F}(M, \mathcal{F})$ in the contravariant case) is called an associated geometric structure on the foliated manifold $(M, \mathcal{F})$.

It is not difficult to see that for a given associated natural bundle $F(M, \mathcal{F})$, associated geometric structures on $(M, \mathcal{F})$ which are foliated subbundles of $F(M, \mathcal{F})$ define holonomy invariant subbundles of $F(N)$ to which they are $\mathcal{U}$-related, i.e. $f_{i}$-related for any $i \in I$. 
Example 6. - 1. A global infinitesimal automorphism of the foliation $\mathcal{F}$ is an associated geometric structure; a base-like form is such a structure as well.

2. Let us consider the bundle $L_{\mathcal{F}}(M)$ of adapted linear frames to the foliation $\mathcal{F}$. It is a reduction of the linear frame bundle $L(M)$ to the structure group $G L(n, n-q)$. The foliation $\mathcal{F}_{L}$ of $L_{\mathcal{F}}(M)$, locally, is given by the following submersion : let $(U, \phi), \phi=\left(\phi_{1}, \phi_{2}\right): U \longrightarrow R^{n-q} \times R^{q}$ be an adapted chart, then the mapping $\widetilde{\phi}: L_{\mathcal{F}}(M) \mid U \longrightarrow L\left(R^{q}\right)$,

$$
\widetilde{\phi}\left(v_{1}, \ldots v_{n}\right)=\left(d \phi_{2}\left(v_{1}\right), \ldots d \phi_{2}\left(v_{n}\right)\right)=\left(d \phi_{2}\left(v_{n-q+1}\right), \ldots d \phi_{2}\left(v_{n}\right)\right)
$$

is a submersion defining $\mathcal{F}_{L}$ over $U$.

Associated $G$-structures are foliated reductions of $L_{\mathcal{F}}(M)$ to groups consisting of matrices of the form $\left(\begin{array}{ll}* & * \\ 0 & A\end{array}\right)$ where $A$ is a matrix of a given Lie subgroup $G_{0}$ of $G L(q)$.

3. A linear connection $w$ in the bundle $L_{\mathcal{F}}(M)$ is given by a section $S_{w}$ of the sheaf $A^{1}\left(L_{\mathcal{F}}(M), E\left(L_{\mathcal{F}}(M), g l(n, n-q)\right)\right)$ of 1 -forms on $L_{\mathcal{F}}(M)$ with values in the associated fibre bundle $E\left(L_{\mathcal{F}}(M), g l(n, n-q)\right.$ ) (to be precise we take the pull-back of this bundle to the total space of $\left.L_{\mathcal{F}}(M)\right)$. Such a connection is an associated geometric structure (or an associated connection) if in the bundle $\mathrm{L}(\mathrm{N})$ there exists a connection $w_{N}$ given by a section $S_{N}$ which is $\mathcal{U}$-related to the $g l(q)$-component of $S_{w}$. Locally, it means that for any submersion $f: U \longrightarrow N$ defining the foliation $\mathcal{F}$ the following diagram is commutative :

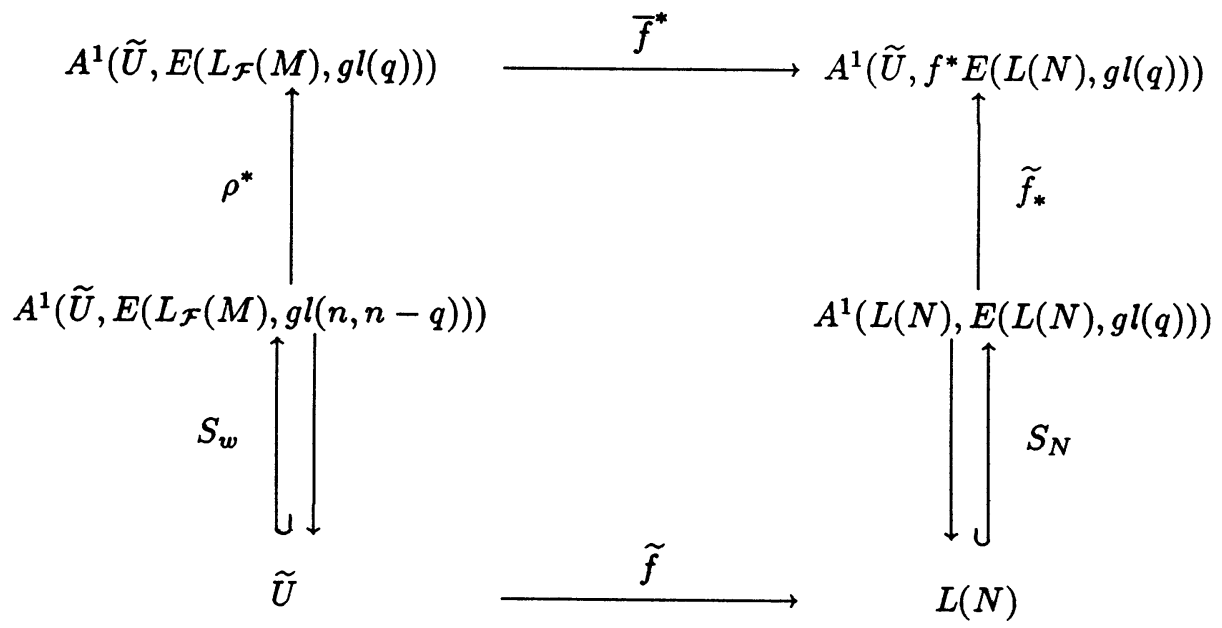


where $\widetilde{U}=L_{\mathcal{F}}(M) \mid U, \tilde{f}$ is the mapping of frame bundles induced by $f$, $\tilde{f}_{*}$ and $\bar{f}$ the corresponding mappings of the sheaves and associated fibre bundles, respectively, and $\rho^{*}$ the mapping induced by the homomorphism $\rho: g l(n, n-q) \longrightarrow g l(q)$ defined by the correspondence $g l(n, n-q) \ni$ $\left(\begin{array}{cc}* & * \\ 0 & A\end{array}\right) \longmapsto A \in g l(q)$. Thus

$$
\bar{f}^{*} \rho^{*} S_{w} \mid \widetilde{U}=\tilde{f}_{*} S_{N} \tilde{f}
$$

and therefore

$$
\rho w=\tilde{f}^{*} w_{N} .
$$

To be absolutely precise, such a connection itself is not an associated structure, but rather a class of connections in $L_{\mathcal{F}}(M)$ having the same $g l(q)$-component.

In terms of geometric properties it means that the parallel transport on $M$ defined by the connection $w$ projects onto the parallel transport on the transverse manifold $N$ defined by the connection $w_{N}$. Such pairs of connections were studied by R.A.Blumenthal in $[9,10]$.

4. Let us consider associated $\{e\}$-structures. An associated $\{e\}$-structure is a foliated section of the bundle $L_{\mathcal{F}}(M)$; thus at each point $x$ we have a linear frame $\left(v_{1}, \ldots v_{n}\right)$ of $L_{\mathcal{F}}(M)$. Moreover, at any two points $x_{1}$ and $x_{2}$ of $U_{i}$ such that $f_{i}\left(x_{1}\right)=f_{i}\left(x_{2}\right)$, the value of the mapping $\tilde{f}_{i}$ on these frames is the same, i.e. $d f_{i}\left(v_{j}\left(x_{1}\right)\right)=d f_{i}\left(v_{j}\left(x_{2}\right)\right)$. Therefore, the global vector fields $X_{j}, X_{j}(x)=v_{j+n-q}(x), j=1, \ldots q$, are infinitesimal automorphisms defining a transverse parallelism of $\mathcal{F}$.

It is easy to verify that on the total space of $L_{\mathcal{F}}(M)$ an associated connection defines an associated $\{e\}$-structure for the foliation $\mathcal{F}_{L}$.

5. Prolongations of associated $G$-structures provide other examples of associated geometric structures; for details on prolongations of $G$-structures see $[20,35]$. Let us consider the vector space $R^{n}$ as the product $R^{p} \times R^{q}, p=$ $n-q$, the natural projection $\rho_{0}: R^{n} \rightarrow R^{p}$ and the natural inclusion $s_{0}: R^{q} \rightarrow R^{n}$. Then let us take the Lie subgroup $G$ of $G L(n, p)$ consisting of matrices of the form $\left(\begin{array}{ll}* & * \\ 0 & A\end{array}\right)$ with $A$ from a Lie subgroup $G^{\prime}$ of $G L(q)$. The correspondence $\rho:\left(\begin{array}{ll}* & * \\ 0 & A\end{array}\right) \longmapsto A$ defines a homomorphism of Lie groups $\rho: G \longrightarrow G^{\prime}$ and of Lie algebras $\rho: g \longrightarrow g^{\prime}$. The mapping $s: g^{\prime} \longrightarrow g$ defined as $s(B)=\left(\begin{array}{ll}0 & 0 \\ 0 & B\end{array}\right)$ is a section of $\rho$. 
Foliated and associated geometric structures on foliated manifolds

It is straight-forward to verify that the mapping $\rho$ induces surjective homomorphisms $\rho_{k}$ of the subsequent prolongations of the Lie algebras of $g$ and $g^{\prime} ; \rho_{k}: g^{(k)} \longrightarrow g^{(k)}$.

As the result of these considerations we obtain the following lemma.

LEMMA 2. - If the Lie algebra $g$ is of type $k$, the Lie algebra $g^{\prime}$ is of type $k^{\prime}, k^{\prime} \leq k$.

Let $f: M \longrightarrow N$ be a surjective submersion. Let $B(M, G, \pi)$ be a $G$ structure on the $n$-manifold $M$ and $B^{\prime}\left(N, G^{\prime}, \pi^{\prime}\right)$ a $G^{\prime}$-structure on the $q$ manifold $N$ which are $f$-related. Then for the fundamental forms $\theta$ and $\theta^{\prime}$ of $B(M, G, \pi)$ and $B^{\prime}\left(N, G^{\prime}, \pi^{\prime}\right)$, respectively, we have the following :

$$
\theta^{\prime} \circ d \tilde{f}=\rho_{0} \circ \theta
$$

Let $X \in T_{p} B$, then $\theta^{\prime}(d \tilde{f}(X))=\widetilde{f}(p)^{-1} d \pi^{\prime}(d \tilde{f}(X))=\tilde{f}(p)^{-1} \circ d f \circ d \pi(X)$. As $(\tilde{f}(p))^{-1} \circ d_{x} f=\rho_{0} p^{-1}$ where $\pi(p)=x$, we have

$$
\theta^{\prime}(d \tilde{f}(X))=\rho_{0} p^{-1} d \pi(X)=\rho_{0} \theta(X) .
$$

We would like to show that the consecutive prolongations of the $f$-related structures $B(M, G, \pi)$ and $B^{\prime}\left(N, G^{\prime}, \pi^{\prime}\right)$ are $f$-related. First of all, we shall demonstrate that their structure tensors are $\tilde{f}$-related.

The structure tensor $c$ of $B(M, G, \pi)$ takes values in

$$
\operatorname{Hom}\left(R^{n} \wedge R^{n}, R^{n}\right) / \partial H o m\left(R^{n}, g\right)=H^{0,2}(g) .
$$

The mappings $\rho$ and $\rho_{0}$ induce the mappings $\hat{\rho}: \operatorname{Hom}\left(R^{n} \wedge R^{n}, R^{n}\right) \longrightarrow$ $H o m\left(R^{q} \wedge R^{q}, R^{q}\right)$ and $\tilde{\rho}: H^{0,2}(g) \longrightarrow H^{0,2}\left(g^{\prime}\right)$, the second one being the quotient of the first. The mappings $s$ and $s_{0}$ define the corresponding sections of $\hat{\rho}$ and $\tilde{\rho}$, respectively, i.e. $\widehat{s}: \operatorname{Hom}\left(R^{q} \wedge R^{q}, R^{q}\right) \longrightarrow \operatorname{Hom}\left(R^{n} \wedge\right.$ $\left.R^{n}, R^{n}\right)$ and $\widetilde{s}: H^{0,2}\left(g^{\prime}\right) \longrightarrow H^{0,2}(g)$. The equality (1) ensures that

$$
c^{\prime} \circ \tilde{f}=\tilde{\rho} \circ c .
$$

Take a subspace $C$ of $H o m\left(R^{n} \wedge R^{n}, R^{n}\right)$ supplementary to $\partial H o m\left(R^{n}, g\right)$ such that the space $C \cap \widehat{s}\left(\partial \operatorname{Hom}\left(R^{q}, g^{\prime}\right)\right)$ is in $\widehat{s}\left(\operatorname{Hom}\left(R^{q} \wedge R^{q}, R^{q}\right)\right)$. Then $\widehat{\rho}\left(\widehat{C^{\prime}}\right)=C^{\prime}$ is supplementary to $\partial H o m\left(R^{q}, g^{\prime}\right)$ in $\operatorname{Hom}\left(R^{q} \wedge R^{q}, R^{q}\right)$.

Let $V$ be a horizontal subspace of $T_{p} B$ such that $c_{V} \in C$. The equality (1) ensures that for the horizontal subspace $V^{\prime}=d \tilde{f}(V), c_{V^{\prime}} \in C^{\prime}$, as $\rho c_{V}=$ $c_{V^{\prime}}$. This implies that the correspondence $V \longmapsto d \tilde{f}(V)=V^{\prime}$ defines a 
transformation $f_{1}$ of the first prolongation $B^{(1)}(M, G, \pi)$ of the $G$-structure $B(M, G, \pi)$ onto the first prolongation $B^{\prime(1)}\left(N, G^{\prime}, \pi^{\prime}\right)$ of the $G^{\prime}$-structure $B^{\prime}\left(N, G^{\prime}, \pi^{\prime}\right) . f_{1}$ covers $\tilde{f}$ and makes these two structures $\tilde{f}$-related, and thus the bundles $B^{(1)} \longrightarrow M$ and $B^{\prime(1)} \longrightarrow N f$-related. Repeating the construction we obtain mappings $f^{k}: B^{(k)}(M, G, \pi) \longrightarrow B^{\prime(k)}\left(N, G^{\prime}, \pi^{\prime}\right)$ covering the mappings $f^{k-1}$ and $f$, and making these structures $f$-related. If the group $G$ is of type $k+1$, the total spaces of the bundles $B^{(k)}(M, G, \pi)$ and $B^{\prime(k)}\left(N, G^{\prime}, \pi^{\prime}\right)$ are parallelisable and these parallelisms are $f^{k}$-related.

In the case of a foliation $\mathcal{F}$ defined by a cocycle $\mathcal{U}$, the above construction yields structures $B^{(k)}(M, G, \pi)$ and $B^{(k)}\left(N, G^{\prime}, \pi^{\prime}\right)$ which are $\mathcal{U}$-related. For a group $G$ of type $k+1$, the foliation $\mathcal{F}_{k+1}$ of the total space $B^{k}$ of the bundle $B^{(k)}(M, G, \pi)$ is transversely parallelisable. The parallelisms of the manifolds $B^{k}$ and $B^{\prime k}$ are $\mathcal{U}_{k}$-related where $\mathcal{U}_{k+1}$ is the cocycle defining $\mathcal{F}_{k+1}$ derived from the cocycle $\mathcal{U}$.

Before we can formulate our main theorem on associated geometric structures we need the following definitions.

Definition 7.- $A$ G-structure $B(M, G, \pi)$ of type $k+1$ is complete if the parallelism of the total space $B^{k}$ of the kth-prolongation $B^{(k)}(M, G, \pi)$ is complete.

DEFINITION 8.- 1. An associated geometric structure $E(M, \mathcal{F})$ is of finite type if the total space $E$ of this bundle is parallelisable and this parallelism is $\mathcal{U}_{E}$-related to a parallelism of the total space of the corresponding bundle on the transverse manifold.

2. An associated geometric structure $E(M, \mathcal{F})$ of finite type is complete if the vector space spanned by the vector fields of the parallelism of $E$ consists of complete vector fields.

3. An associated geometric structure $E(M, \mathcal{F})$ is a Serre structure if the projection in the bundle $E(N)$ is a Serre fibration.

Example 7. - Associated $G$-structures of finite type and $G$-structures of higher order, cf. [31,11], are Serre associated geometric structures of finite type.

THEOREM 6. - Let the foliation $\mathcal{F}$ be an $(N, K)$-structure on a manifold $M$. If $(M, \mathcal{F})$ admits a complete Serre associated geometric structure $E(M, \mathcal{F})$ of finite type, then the natural projection $p: \widetilde{M} \longrightarrow \widetilde{M} / \widetilde{\mathcal{F}}$ of the universal covering space $\widetilde{M}$ of $M$ onto the space of leaves $\widetilde{M} / \widetilde{\mathcal{F}}$ of the lifted 
foliation $\widetilde{\mathcal{F}}$ is a Serre fibration and the space $\widetilde{M} / \widetilde{\mathcal{F}}$ is a Hausdorff manifold.

Proof.- From the very beginning we can assume that the manifold $N$ is simply connected. We can always take its universal covering and the group generated by the action of $K$ on it. Since elements of $K$ lift to diffeomorphisms preserving the parallelism of the total space of $E(N), K$ acts quasi-analytically on $N$. Therefore $\mathcal{F}$ is developable. Let $n: \widetilde{M} \longrightarrow N$ be the developing mapping. Then we have the following commutative diagram :

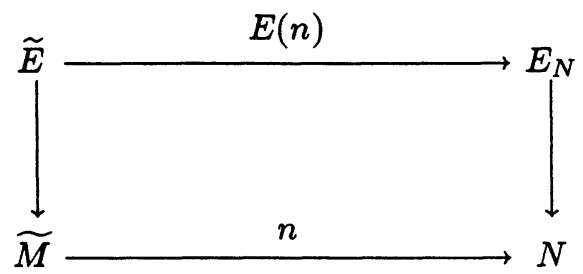

where $\widetilde{E}(\widetilde{M}, \widetilde{\mathcal{F}})$ is the lift of the bundle $E(M, \mathcal{F})$ to $\widetilde{M}$. The parallelisms of the total spaces $\widetilde{E}$ and $E_{N}$ of the bundles $\widetilde{E}(\widetilde{M}, \widetilde{\mathcal{F}})$ and $E(M, \mathcal{F})$ are $E(n)$ related. Therefore, the lifted foliation $\widetilde{\mathcal{F}}_{E}$ of $\widetilde{E}$ which is defined by the global submersion $E(n)$ is a complete transversely parallelisable foliation. Thus the submersion $E(n)$ is a locally trivial fibre bundle, and the developing mapping is surjective. The fact that the projection $E_{N} \longrightarrow N$ is a Serre fibration ensures that the developing mapping itself is a Serre fibration. Since the manifold $N$ is simply connected, its fibres must be connected and the space of leaves of the foliation $\widetilde{\mathcal{F}}$ is just the manifold $N$.

Remarks. - This theorem generalizes results of R.A.Blumenthal contained in $[9,10,11]$. In its main outline the proof is the same as the one presented by him and can be considered as a kind of "proof scheme". By adopting various assumptions on geometric structures (as we have done in section 2) and imposing the completeness conditions we can prove a series of results on associated geometric structures which correspond to theorems proved in the previous section. It is worth stressing that the completeness of an associated structure does not imply the completeness of the corresponding foliated structure.

This kind of theorems is quite useful in the study of homotopy and homology groups of leaves and their relations with the corresponding groups of the ambient manifold, cf. $[9,10,11]$. As an example we can give the following corollary. 
COROLlarY 4.- Let the model space $N$ be contractible and the holonomy pseudogroup equivalent to the one generated by a group $K$. Then for any leaf $L$ of the foliation $\mathcal{F}$ the homotopy groups $\pi_{i}(L)$ inject into the corresponding homotopy groups of the ambient manifold $M$.

\section{Références}

[1] Albert (C.), Molino (P.). - Pseudogroupes de Lie transitifs, Travaux en Cours, Hermann, Paris, 1984 .

[2] Amores (A.M.). - Vector fields of finite type $G$-structures, J. Diff. Geom. 14, 1979, p. 1-6.

[3] Blumenthal (R.A.). - Foliated manifolds with flat basic connections, J. Diff. Geom. 16, 1981, p. 401-406.

[4] Blumenthal (R.A.). - Basic connections with vanishing curvature and parallel torsion, Bull. Sci. math. 106, 1982, p. 393-400.

[5] Blumenthal (R.A). - Riemannian foliations with parallel curvature, Nagoya Math. J. 90, 1983, p. 145-153.

[6] Blumenthal (R.A). - Foliations with locally reductive normal bundle, Illinois J. Math. 28, 1984, p. 691-702.

[7] Blumenthal (R.A). - Stability theorems for conformal foliations, Proc. A.M.S. 91,1984 , p. 55-63.

[8] Blumenthal (R.A). - Cartan connections in foliated bundles, Michigan Math. J. 31,1984 , p. $55-63$.

[9] Blumenthal (R.A). - Submersions affines et feuilletages de variétés munies d'une connexion linéaire, C. R. Acad. Sc. Paris 299, 1984, p. 1013-1015.

[10] Blumenthal (R.A). - Affine submersions, Ann. Glob. An. Geom. 3, 1985, p. 275287.

[11] Blumenthal (R.A). - Cartan submersions and Cartan foliations, Illinois J. Math. $31,2,19^{8} 7$, p. 327-343.

[12] Carrière (Y.), Ghys (E.). - Feuilletages totalement géodésiques, An. Acad. Brasil Ciênc. 53, 1981, p. 427-432.

[13] Epstein (D.B.A.). - Foliations with all leaves compact, Ann. Inst. Fourier 26, 1, 1976, p. 265-273.

[14] Goldman (W.), Hirsch (M.W.), Levitt (G.). - Invariant measures for affine foliations, Proc. AMS 86, 1982, p. 511-518.

[15] HAEfliger (A.). - Structures feuilletées et cohomologie à valeurs dans un faisceau de groupoïdes, Comm. Math. Helv. 32, 1958, p. 248-329.

[16] Haefliger (A.). - Pseudogroups of local isometries, Differential Geometry, L.A.Cordero ed., Proceedings Vth International Colloquium on Differential Geometry, Santiago de Compostela 1984, Pitman 1985.

[17] Haefliger (A.). - Leaves closures in Riemannian foliations, A fête of topology, Academic Press, Boston, MA, 1988, p. 3-32. 
Foliated and associated geometric structures on foliated manifolds

[18] Kamber (F.W.), Tondeur (Ph.). - Characteristic Classes and Foliated Bundles, Springer Lecture Notes in Math. 493, 1975.

[19] Kamber (F.W), Tondeur (Ph). - G-foliations and their characteristic classes, Bull. A.M.S. 84, 6, 1978 , p. 1086-1124.

[20] Koваyashi (S). - Transformation Groups in Differential Geometry, Springer, Berlin, 1972.

[21] Kobayashi (S.), Nomizu (K.). - Foundations of Differential Geometry, Interscience Publ., New York 1963, 1969.

[22] Ledger (A.Y.), ОвAta (M). - Compact Riemannian manifolds with essential groups of conformorphisms, Trans. A.M.S 150, 1970, p. 645-651.

[23] Libermann (P.). - Pfaffian systems and transverse differential geometry, Differential Geometry and Relativity, D.Reidel, 1976, p. 107-126.

[24] Molino (P.).-Connexions et $G$-structures sur les variétés feuilletées, Bull. Sc. math. 92, 1968, p. 59-63.

[25] Molino (P.). - Propriétés cohomologiques et propriétés topologiques des feuilletages à connection transverse projetable, Topology 12, 1973, p. 317-325.

[26] Molino (P.). - Sur la géométrie transverse de feuilletages, Ann. Inst. Fourier 25, 1975, p. 279-284.

[27] Molino (P.). - Le problème d'équivalence pour les pseudogroupes de Lie, méthodes intrinsèques, Bull. Soc. Math. France 108, 1980, p. 95-111.

[28] Molino (P.). - Invariants structuraux des feuilletages, Bull. Sc. math. 105, 1981, p. 337-347.

[29] Molino (P.). - Riemannian Foliations, Progress in Math., Birkhäuser, 1988.

[30] Nomizu (K.). - On local and global existence of Killing vector fields, Ann. of Math. 72, 196o, p. 105-120.

[31] OснаI (T.).- Geometry associated with semisimple flat homogeneous spaces, Trans. A.M.S. 152, 1970, p. 159-193.

[32] Palais (R.S.), Terng (C.L.). - Natural bundles have finite order, Topology 16, 1977, p. 271-277.

[33] RASMUSSEN (O.H.). - Foliations with integrable transverse $G$-structures, J. Diff. Geom. 16, 1981 , p. 699-710.

[34] Rodriguez (A.M.). - The first and second fundamental theorems of Lie for Lie pseudogroups, Amer. J. Math. 84, 1962, p. 265-282.

[35] Sternberg (S.). - An Introduction to Differential Geometry, Chelsea Publ.C. (second edition), New York 1983 .

[36] TAKEUCHI (M.).- On foliations with the structure group of automorphisms of a geometric structure, J. Math. Soc. Japan 32, 1980, p. 119-152.

[37] Thurston (W.). - Topology of 3-Manifolds, Princeton.

[38] Wolf (J.A.). - Spaces of Constant Curvature, Publish or Perish Inc., 1974.

[39] Wolak (R.). - On $\nabla-G$-foliations, Suppl. Rend. Cir. Mat. Palermo 6, 1984, p. 317-325.

[40] Wolak (R.). - On G-foliations, Ann. Pol. Math. 46, 1985, p. 329-341. 
[41] Wolak (R.). - On transverse structures of foliations, Suppl. Rend. Cir. Mat. Palermo 9, 1985, p. 22i-243.

[42] WolaK (R.).- Foliations admitting transverse systems of differential equations, Comp. Math. 67, 1988, p. 89-101.

[43] WolaK (R.). - Transverse completeness of foliated systems of ordinary differential equations, Proc. VIth Inter. Coll. on Differential Geometry, Santiago de Compostela 1988, ed. L. A. Cordero, Santiago de Compostela 1989.

[44] WoLAK (R.). - G-foliations of finite type with all leaves compact, to be publ. in Publ. Mat. UAB, 1988.

(Manuscrit reçu le 4 octobre 1988) 